Pediatr. Res. 16: 1049-1051 (1982)

\title{
Author Index to Volume 16
}

Abdul Rahiman M 663

Adebonojo FO 982

Aicardi J 259

Akamatsu $\mathrm{H} 5$

Aladjem M 840

Alkonyi I 89

Allan L 126

Alloisio N 1016

Alvarez JR 728

American Academy of Pediatrics Council 264

American Pediatric Society Council 264

Andersen DW 304

Anderson GH 65

Angle CR 331

Anstall H 57

Aoki T 5

Aperia A 351

Arakawa, K 247

Ariagno RL 512

Arnaud MJ 167

Arthus MF 684

Assael BM 810

Auconi P 1022

Auricchio S 1004

Avendano LF 329

Awasthi YC 21

Back J-F 92

Bagnoli F 95

Bahna SL 604

Baley JE 649

Balistreri WF 282

Barajas L 424

Bargen M 846

Baricos WH 933

Barrett AJ 613

Barrett C 869

Barnett DR 21

Barnett HL 807

Bassi JA 746

Battaglia FC 310, 716

Baum D 995

Bellanti JA 263

Bennett M 286

Benoit O 299

Bensman A 92

Benson PF 260

Bergeron M 684

Berggren P 92 I

Berman W 357

Berry HK 751

Besmond C 335

Bezou MJ 412

Bhutani VK 830

Biagini R 1022

Bidani A 277

Blanc WA 109

Blackberg L 882

Bland RD 583

Blitzer MG 203, 938
Boat TF 181,792

Bofinger MK 751

Bogart BI 223

Borrone C 954

Bossi E 579

Boucek RJ 721

Bougle D 400

Boule M 779

Bourbon J 50

Bowles B 192

Bowman BH 13, 21

Bracci R 95

Bracco I 167

Bradley PP 57

Brandt NJ 861

Brans YW 989

Brass CA 388

Bratteby L-E 152, 156

Braun LD 324

Briggman JV 1035

Broyer M 354

Brunser O 187

Buehring GC 460

Burgoyne R 834

Burkett ES 632

Bury AF 613

Businco L 1000

Button LR 260

Calcagno PL 340

Calderon A 329

Candee D 846

Carlsson B 272

Carr BR 682

Carson SD 13

Castleberry RP 484

Cavanna G 810

Chan HSL 474, 477

Chantler C 784

Charpentier C 85

Chen J 130

Cheng PW 792

Chevalier RL 436

Chui D 928

Chitra, CI 638

Christensen E 861

Christensen MF 861

Christensen RD 57, 549

Chuck G 192

Churchill P 277

Clarke WR 8, 163, 538, 732

Clayton, GW 118

Coates PM 982

Cohen HJ 373

Colarizi P 1022

Compton SK 561

Cook CD 846

Cordero ME 187

Corey M 554

Corke BC 621

Cornford EM 324

Cortner JA 982
Cote J 137

Cotton RB 35

Cottreau D 199

Coude FX 85, 877

Coulet M 412

Coulombe P 238

Cox F 816

Crawford J 314

Crist WM 484

Crokaert R 761

Cruz JR 272

Cuezva JM 632, 638

Cunnane SC 599

Curley K 370

Curstedt T 921

Dab I 761

Daegelen D 335

Dakshinanurti K 126

Dalens B 412

Dardenne M 92

Darnall RA Jr 512

Darovec-Beckerman C 381

Davies EG 573

Dean MF 260

Deal CL 820

Dechaux M 354

Delaunay J 1016

Del Principe D 1000

Demaugre F 877

Denning CR 223

de Ritis G 1004

DeVane GW 504

De Vincenzi M 1004

Domenech E 675

Dorleac E 1016

Dosik H 769

Downes JJ 290

Dreyfus JC 335

Durand P 954

Duarte E 329

Dubin JW 653

Durie PR 554

Dussault JH 238

Eisner GM 340

Elde RP 446

Elinder G 351

Endo F 227

Enhorning G 834

Erenberg A 570

Eto Y 395

Eveleth D 520

Facchinetti F 95

Fannon L 35

Felder RA 340

Feldmann, G 441

Fielek S 960

Fiezal J 259

Filer LJ 304

Finklestein J 798
Fischer DP 499

Fischer JM 499

Fisher DA 520, 569

Fleming PJ 1031

Fomon SJ 161

Fondacaro JD 494

Fontaine J-L 441

Forstner GG 554

Fox WW 290

Francois B 977

Frank L 570

Frazier JP 558

Freedman MH 474, 477

Freier S 69

Frisch A 232

Gaerlan PA 223

Galant SP 591

Galey WR 104

Galli E 1000

García B 272

Garry PJ 161

Gaskin KJ 554

Gaultier CL 779

Gebre-Medhin M 272

Gelfand E 362

Genazzani AR 95

Gewolb IH 869

Ghishan FK 295, 566

Ghishan FK 776

Gibson KM 877

Gilbert M 310

Gillon MJ 104

Girard F 779

Girard J 310, 716

Glatz T 424

Glatz TH 711

Gleason WA 403

Goad WB 343

Goedde HW 806

Goeringer GC 561

Goldsmith DI 840

Goldstein E 209

Goldstein R 69

Goncalves A 1031

Gordon BA 217

Gourmelen M 299

Graeber JE 490

Gravel RA 126

Gray B 181

Green RS 35

Greene H 266

Greengard O 388

Gregersen N 861

Gregory G 137

Griffith BP 455

Groothuis DR 499

Grossmann G 921

Grutters G 716

Grylack L 509

Guandalini S 99

Gudinchet F 1025 
Guifoile MB 751

Guilhaume A 299

Guthrie RD 30

Guyda HJ 820

Haberer JP 412

Haberkern CM 30

Hall B 251

Hall D 113

Hall PK 416

Handa T 395

Hansen TN 583

Hanson LÅ 272

Hart GH 286

Hartemann D 400

Hashimoto T 430

Hatch DJ 943

Hayasaka K 5

Hechtman P 217

Hedner J 543

Hedner T 543

Heiner DC 604

Heller RH 232

Hellmann $\mathbf{J} 40$

Helms PJ 943

Heng EL 400

Hernell O 45, 882

Herschkowitz NN 395, 579, 588

Heubi JE 494

Heymann MA 594, 705

Hill HR 347, 549

Hilty MD 176

Hine DG 740

Hiraga K 5

Hirschhorn K 917

Hirschhorn R 362

Hodes HL 920

Hofvander Y 272

Holcombe JH 118

Holman RT 45

Holmberg L 256

Holmgren G 45

Hoo JJ 806

Hors-Cayla MC 259

Hsieh HL 933

Hsiung GD 455

Hug A 769

Hug G 192

Hughes WT 874

Hull D 60

Hulse MG 943

Hunt MM 751

Hunter MA 989

Husted SDR 846

Ii $\mathrm{K} 430$

Ikegami M 424, 711

Isaacs CE 388

Ise I 247

Ito $\mathrm{M} 430$

Iwasumi T 21

Jaccoud P 1016

Jacobs H 424

Jagell SF 45

Janas LM 659

Jenkins MQ 1035

Jequier $E 1025$

Jobe A 424, 711

Johnson C 877

Johnson DE 446

Johnson HJ Jr 682
Johnson S 45

Jonason J 543

Jones JF 525

Jones RWA 784

Jones SJ 424

Jose PA 340

Jost A 50

Kaapa P 827

Kahn A 199, 335

Kalhan SC 608

Kalnitsky A 628

Kameyama J 771

Kang SS 370

Karayalcin G 318

Kawakami T 5

Kazazian HH 232

Keenan BS 118

Keller MA 604

Kellogg FW 494

Kerner J 89

Kerr DS 632

Kesarwala H 362

Kettrick RG 290

Kim O 470

King KC 608

Kirkland RT 118

Kisker CT 8, 538, 732

Kleinman LI 148

Knauss TA 30

Kobayashi Y 243

Koblin BA 874

Kogut MD 1

Kohl S 558

Koivisto M 827

Kolodny EH 232

Kolvraa S 861

Komnick K 756

Kosugi E 247

Kotagal UR 148

Krantz B 141

Kuipers JRG 594, 705

Kuroda Y 430

Lachmann B 921

Lancaster GA 86

Landrieu P 977

Lankford GL 403

Lanzkowsky P 318

Lasfargues G 92

Lauer RM 163

Leake RD 569

Lebenthal E 141, 470

Lee PC 470

Leeper L 295

Lemons JA 113

Lenoir G 354

Leung CCK 973

Levinsky RJ 573

Levine MR 1031

Levy E 69

Li PK 141

Liechti-Gallati S 588

Limal JM 354

Lindberg T 479

Linderkamp O 964

Lindstrom DP 35

Lippert W 209

Ljung R 256

Lock JE 446

Logvinoff MM 969
London JW 644

Lonnerholm G 407

Loo LS 558

Lubin B 928

Lucia HL 455

Lundberg D 543

Lyon G 977

Macaya J 329

MacDonald PC 682

Macfarlane JL 549

Madahar C 769

Mahoney LT 163

Mahoney MJ 740

Malluh A 484

Mandelbaum I 761

Mandyla H 995

Mann DR 176

Maravelias C 995

Marchal F 621

Margolies R 181

Margueritte G 1016

Marie J 335

Mark AL 163

Marone PA 109

Maroteaux P 259

Marsac C 85

Marsh GW 286

Maruyama S 377

Matsaniotis N 995

Matsuda I 227

Matsuo N 5

Matty PJ 653

Matz S 463

Mayumi M 247

McChesney R 388

McCoy EE 314

McIntire MS 331

McKiernan J 60

McNeely MC 21

Medow MS 644

Meiselman HJ 964

Meneely R 295, 566, 776

Menichelli A 1000

Merritt TA 798

Metcalfe IL 834

Metoki K 5

Micheli H 665

Micheli JL 1025

Migeon BR 172

Miller CS 232

Miller F 318

Miller K 649

Mills A 137

Milner RDG 213

Mischler E 416

Miyakawa Y 247

Miyao M 430

Moessinger AC 109

Mohrenweiser HW 960

Momoi T 771

Moore JJ 381

Morle L 1016

Morray JP 290

Morriss FH Jr 851

Moser AB 172

Moser H 588

Moser HW 172

Moya M 675

Moye L 113

Mozes N 761
Muir H 260

Muller DPR 251

Munnich A 85, 259, 335

Murthy PB 663

Muzzo S 187

Naden RP 504

Naito S 247

Nardis EE 40

Narisawa K 5

Nelles LP 416

Neufeld EF 232

Neugebauer D 509

Newburger PE 373, 856

Nezelof C 199

$\mathrm{Ng}$ Ying Kin NMK 217, $53 \mathrm{C}$

Nitowsky HM 172

Norkus EP 746

Norum RA 172

Notter RH 130, 515

Nyhan WL 877

Oberhansli I 665

O'Brien JS 232

Occorsio P 1004

Ogata A 227

Ogier H 85, 259

Ogra PL 266

Ohlesson K 479

Oldendorf WH 324

Olsson T 35

Opioids 95

Pabst H 314

Papageorgiou P 362

Parini R 810

Parker CR Jr 682

Parmley RT 484

Pascali V 1022

Pasternak JF 499

Patel MS 632, 638

Patton CJ 1035

Pecsuvac K 89

Peeters LLH 716

Perlini R 1000

Perret L 779

Persinai M 1000

Philippart M 954

Philippe N 1016

Philipps AF 653

Phillips PJ 751

Picarelli J 223

Picciano MF 659

Pickering LK 558

Pittard WB 649

Poenaru L 259

Pollinger J 381, 463

Polony I 792

Polsen DC 109

Pometta D 665

Porter JC 504

Posner BI 820

Potts E 118

Powers JM 172

Prchal JT 484

Prenzel I 329

Priebat DA 57

Puck MH 343

Qazi Q 769

Quinton PM 533 
Raghavan S 232

Rasmussen K 861

Ratech H 362

Raye J 653

Raymond L 665

Reichart E 400

Reynolds LW 232

Rhodes ML 570

Richardet JM 299

Rigden SP 784

Roan Y 591

Roberts RC 416

Roberts RJ 570

Robertson B 921

Robillard JE 8, 732

Robinson A 343

Robinson HMP 1010

Rodman HM 632

Roe TF 1

Roegner-Maniscalco V 362

Rogiers V 761

Rojas J 35

Rosenblatt DS 628

Rosenfeld CR 504

Rosenstein BJ 874

Roses AD 589

Rossi P 1000

Rosso P 746

Roth KS 126, 644

Rothstein G 57, 549

Rousseau-Merck M 199

Rubino A 99

Rubinstein A 362

Rudolph AM 594, 705

Ruel J 238

Rusconi F 810

Ruuskanen O 649

Sachs C 354

Saida K 377

Saik LA 933

Salle BL 75

Salvesen GS 416

Sandor A 89

Santos Jl 347

Sato $T 377$

Satoh T 5
Saudubray JM 85, 259, 877

Saunders EF 474, 477

Saunders M 126

Scanlon JW 509

Schaumburg HH 172

Schneider L 21

Schulman JD 490

Schutz Y 1025

Scriver CR 86, 684

Seakins A 1010

Serabian MA 644

Shaffer TH 830

Shafrir E 69

Shannon DL 989

Shapira E 203, 933, 938

Shapiro DL 130

Shapiro J 232

Sheehan TJ 846

Sherman M 209

Shiffman ML 104

Shigematsu Y 771

Shigeoka AO 347

Shortencarrier R 357

Sidi D 594, 705

Siegel M 141

Siegrist HP 579, 588

Silano V 1004

Simmons M 113

Simpson ER 682

Skovbjerg H 948

Slott JH 490

Smith C 515

Sobonya RE 969

Society for Pediatric Research Council 264

Sparks J 310

Sparks JW 716

Spedale SB 851

Spicer SS 1035

Spitzer A 840

Srivastava SK 21

Stahlman MT 35

Stefaniak J 123

Stegink LD 304

Stettler E 1025

Stohs SJ 331

Strecker G 259

Stroop S 566
Strynadka K 314

Stuart MJ 490

Suchy FJ 282

Sudo M 771

Suenram A 665

Sundell H 621

Suzuki Y 771

Swanson MS 331

Swanström S 152, 156

Tada K 5

Takahashi K 247

Takata $\ 377$

Tan KL 670

Tanaka K 740

Tanaka S 227

Tannous R 538

Tateda H 5

Tauber AI 856

Taubold R 130, 515

Taussig LM 969

Taylor NL 549

Theriault A 969

Thomas GH 232

Thompson. TR 446

Thornton CJ 989

Tokoro T 395

Topper W 141

Torres-Pinedo R 728

Toshima K 430

Tournier G 779

Trauner DA 950

Tserng KI 608

Tsuda F 247

Tulpule PG 663

Tweed WA 137

Ulane RE 490

Urrutia JJ 272

Usui $\Upsilon 243$

Valleteay de Mouillac J 92

Van Hoof F 977

Vannucci RC 40

Vasmant D 441

Verma RS 769

Vert P 400
Vertongen F 761

Viinikka L 827

Vis HL 761

Wade JG 137

Walker P 520

Warshaw JB 869, 911

Watanabe T 430

Waters CB 569

Weatherall DJ 286

Webber ML 343

Wei P 554

Weichsel ME Jr 520

Weinberg JE 569

Weisbrodt NW 851

Welsch C 167

Wennberg R 209

Werlin SL 123

Wertz RL 589

Westrom B 479

White LB 304

Whitsett JA 381, 463

Weismann U 579, 588

Wilson CM 869

Wilson DP 118

Wilson R 82

Wintzensen $\mathrm{H} 861$

Wirdnam PK 213

Wistrand P 407

Wolfe LS 530

Wong PWK 370

Wood WG 286

Woodrum DE 30

Wu PYK 964

Wu-Rideout MYC 304

Xanthou M 995

Yabek SM 357

Yang W 126

Ylikorkala O 827

Younoszai MK 756

Zinn AB 740

Zlotkin S 65, 628

Zuppinger K 579

Zvaighaft A 187 